\title{
Comparing COVID-19 Critical Care Admissions by Minority Populations to Pre-COVID-19 Critical Care Utilization
}

Panagis Galiatsatos ( $\sim$ panagis@jhmi.edu )

Johns Hopkins University

Kathleen Page

Johns Hopkins: Johns Hopkins University

Souvik Chatterjee

Johns Hopkins: Johns Hopkins University

Joyce Maygers

Johns Hopkins: Johns Hopkins University

Sauradeep Sarkar

Sinai Health System: LifeBridge Health

Vanya Jones

Johns Hopkins: Johns Hopkins University

Tina Tolson

Johns Hopkins: Johns Hopkins University

Felicia Hill-Briggs

Johns Hopkins: Johns Hopkins University

Edward Chen

Johns Hopkins: Johns Hopkins University

Sherita Golden

Johns Hopkins: Johns Hopkins University

\section{Research article}

Keywords: COVID19, Critical Care Utilization, Health Disparities

Posted Date: November 9th, 2020

DOI: https://doi.org/10.21203/rs.3.rs-102318/v1

License: (a) This work is licensed under a Creative Commons Attribution 4.0 International License.

Read Full License 


\section{Abstract}

Background: Several months into the COVID-19 pandemic, reassessing intensive care unit (ICU) utilization, specifically with regional impact on diverse populations, should be a priority for hospitals planning for critical care resource allocation. In our study, we reviewed the impact of COVID-19 on a community hospital serving an urban region, comparing the sociodemographic distribution of ICU admissions before and during the pandemic.

Methods: We executed a time sensitive analysis to see if COVID-19 ICU admissions reflect regional sociodemographic populations as well as ICU admission trends prior to the current pandemic. Collected sociodemographic variables included sex, race, ethnicity, and age of adult patients (age 18 and older) admitted to the hospital's medical and cardiac ICUs, which were converted to COVID-19 ICUs. The time period selected was 18-months, which was then dichotomized into pre-COVID-19 admissions (December 1, 2018 to March 13, 2020) and COVID-19 ICU admissions (March 14, 2020 to May 31, 2020). Variables were compared using Fisher's exact tests and Wilcoxon tests when appropriate.

Results: During the 18-month period, 1861 patients were admitted to the aforementioned ICUs. The mean age of the 1861 patients was $62.75 \pm 15.57$ years old, with the majority of these patients being male (52.23\%), White (64.43\%), and non-Hispanic/Latinx (95.75\%). There were differences in racial and ethnic distribution comparing pre-COVID-19 admissions to the COVID-19 admissions. Compared to pre-COVID19 ICU admissions, there was an increase in African American versus White admissions ( $p=0.01)$ and an increase in Hispanic/Latinx versus non-Hispanic/Latinx admissions $(p<0.01)$, during the COVID-19 pandemic.

Discussion: During the first three months of admissions to COVID-19 ICUs, there was a rise in admissions among Hispanic/Latinx and African-American patients, while non-Hispanic/Latinx and White patient admissions declined compared to the previous pre-COVID year. These findings support development of strategies to enhance allocation of resources to bolster novel, equitable strategies to mitigate the incidence of COVID-19 in minority populations.

\section{Background}

Optimizing the utilization of intensive care units (ICUs) is a significant healthcare priority in the United States. ${ }^{1}$ This optimization requires a critical understanding of variables that contribute to regional usage of adult ICUs. For instance, geographic and sociodemographic factors (e.g. prevalence of minority race and ethnicity, poverty, educational status) ${ }^{2,3}$ and the presence of certain medical conditions $\mathrm{s}^{4,5}$ are associated with adult ICU utilization and admissions ${ }^{5,6}$. When assessing common diagnoses present on admission to adult medical ICUs, such as sepsis and acute respiratory distress syndrome (ARDS), these diagnoses cluster in regions with high prevalence of certain medical conditions (e.g. hypertension and diabetes) $)^{7}$ and with socioeconomic factors (e.g. race, ethnicity, poverty $)^{5,8}$. Insight into the 
aforementioned variables would ensure efficient use of critical care resources for hospitals, communities, and patients.

The current pandemic caused by the severe acute respiratory syndrome coronavirus-2 (SARS-CoV-2) has created unprecedented demands on acute health care, necessitating the development of triage algorithms typically assigned to battlefields to determine who receives medical treatment or access to medical equipment, and in which order. ${ }^{9}$ Specifically, SARS-CoV-2 resulting coronavirus infectious disease 2019 (COVID-19) frequently results in sepsis and ARDS ${ }^{10,11}$, which warrant ICU admissions. Having reached a point several months into the pandemic, reassessing ICU utilization, specifically regional impact, should be a priority for hospitals in an effort to assist in ongoing public health policies to mitigate the spread of the virus as well as planning for critical care resource allocation.

In our study, we reviewed the impact of COVID-19 on a community hospital serving an urban region. Specifically, we executed a time sensitive analysis to determine if COVID-19 ICU admissions reflect regional sociodemographic populations as well as ICU admission trends prior to the current pandemic.

\section{Methods}

\section{Patient Population}

We obtained 18-months of admissions (December 2018 to May 2020) to the medical intensive care unit (MICU) and the cardiac intensive care unit (CICU) at Johns Hopkins Bayview Medical Center (JHBMC). JHBMC is one of three academic hospitals of the Johns Hopkins Health System, located in Baltimore City and has a potential capacity of 500 beds. The reason for choosing MICU and $\mathrm{CICU}$ is that both were converted to specialized COVID-19 intensive care units (ICU) to provide care for critically-ill patients confirmed to have COVID-19 infection and persons under investigation (PUI). We reviewed all adult patients (18 years or older) admitted to the intensive care units. The study was approved by Institutional Review Board at Johns Hopkins School of Medicine and all actions undertaken by the authors were in accordance with the Declaration of Helsinki.

JHBMC serves diverse populations in community statistical areas of Baltimore City that are within the hospital's proximity (ZIP Codes: 21202, 21205, 21206, 21213, 21218, 21219, 21222, 21224, and 21231). Percentage of African Americans in these regions ranges from 12.4-27.5\%, while percentage of White ranges from $55.2-80.1 \%$. Hispanic/Latinx persons range from $17.1-31.9 \% .{ }^{12}$ Median household income ranges from $\$ 32,102$ to $\$ 71,660$, while the family poverty rate ranges from $15.2-43.6 \%{ }^{12}$

\section{Time Period}

An 18-month review of data for this investigation provides an entire fiscal year of ICU admissions, allowing secular trends to be identified in how specific populations utilized critical care resources over a specific time. In addition, by capturing the first three months of 2020 , we could assure that trends 
observed between March to May 2020 were a) not specific to the current year and b) were not typical seasonal trends (as our data captures March to May 2019).

The 18-month data were then dichotomized into pre-COVID-19 admissions (December 1, 2018 to March 13, 2020) and COVID-19 ICU admissions (March 14, 2020 to May 31, 2020). March 14, 2020 was chosen because it was the first reported case of COVID-19 in Baltimore City ${ }^{13}$ and this timeframe correlated with an increase in COVID-19 admissions in our hospital.

\section{Variables of Interest}

We collected individual sociodemographic data including age, sex, race, ethnicity, and preferred language spoken (identified as "English not preferred") of all patients admitted to the MICU and CICU during the aforementioned timeframe. We defined elderly as age 65 years and older. We also documented length of stay in the ICU as well as ICU-mortality.

\section{Statistical Analysis}

All continuous variables are presented as mean \pm standard deviation or median (interquartile range). Categorical variables were summarized as counts and percentages. Variables were compared using Fisher's exact tests and Wilcoxon tests when appropriate. For comparisons of more than two groups, an analysis of variance (ANOVA) was performed. Conditional density plots were utilized to display population admissions over the 18-month period. Statistical analyses were conducted with R software (V.0.99.903).

\section{Results}

Over the course of 18 months, the MICU and CICU saw a total of 1861 admissions. The mean age of the 1861 patients was $62.75 \pm 15.57$ years (range 19 to 100 years). The majority of these patients were male (52.23\%), White (64.43\%), and non-Hispanic/Latinx (95.75\%). Of the 1861 patients, $419(22.51 \%)$ of them did not survive their hospitalization. A complete list of sociodemographic and hospital variables is provided in Table 1. 
Table 1

Demographic data of the patient population over 18 months. Pre-COVID-19 admissions occurred from December 1, 2018 to March 14, 2020, while post-COVID-19 Admissions occurred from March 15, 2020 to May 31, 2020.

\begin{tabular}{|lllll|}
\hline & $\begin{array}{l}\text { Total } \\
(\mathbf{N}=1861)\end{array}$ & $\begin{array}{l}\text { Pre-COVID-19 } \\
\text { Admissions } \\
(\mathbf{N}=1651)\end{array}$ & $\begin{array}{l}\text { Post-COVID-19 } \\
\text { Admissions } \\
\mathbf{( N = 2 1 0 )}\end{array}$ & $\begin{array}{c}\text { p- } \\
\text { value }\end{array}$ \\
\hline Age (years) & $62.72 \pm 15.57$ & $62.91 \pm 15.42$ & $61.28 \pm 16.65$ & 0.18 \\
\hline Male (\%) & $972(52.23)$ & $857(51.91)$ & $115(54.76)$ & 0.48 \\
\hline Race (\%) & $547(29.39)$ & $473(28.65)$ & $74(35.24)$ & 0.01 \\
African American & $1199(64.43)$ & $1087(65.84)$ & $112(53.33)$ & - \\
White & $115(6.18)$ & $91(5.51)$ & $24(11.43)$ & $<0.01$ \\
Other & & & & \\
\hline Hispanic/Latino (\%) & $79(4.25)$ & $51(3.09)$ & $28(13.33)$ & $<0.01$ \\
\hline English not preferred (\%) & $79(4.25)$ & $52(3.15)$ & $27(12.86)$ & $<0.01$ \\
\hline $\begin{array}{l}\text { ICU Length of Stay } \\
\text { (days) }\end{array}$ & $2.00(1.00$, & $3.00(1.00,5.00)$ & $2.00(1.00$, & 0.95 \\
\hline Death (\%) & $5.00)$ & $367(22.23)$ & $5.75)$ & 0.46 \\
\hline
\end{tabular}

Of the 1861 patients, 210 were admitted between mid-March 2020, after the first case of COVID-19 in Baltimore City, and May 2020. In comparing the pre-COVID-19 admissions to the COVID-19 admissions, there was no statistically significant difference in age $(p=0.18)$, ICU length of stay $(p=0.95)$, or death ( $p$ $=0.46$ ). However, there were differences in the racial and ethnic distribution comparing the pre-COVID-19 admissions to the COVID-19 admissions. For COVID-19 compared to pre-COVID ICU admissions, the increase in African American admissions, as compared to White admissions, was statistically significant $(p=0.01)$ as was the increase in Hispanic/Latinx patients compared to non-Hispanic/Latinx patients $(p<$ 0.01 ) (Table 1). Table 1 further dichotomizes sociodemographic variables by pre-COVID-19 admissions and COVID-19 admissions.

\section{Hispanic/Latinx Patients}

Among Hispanic/Latinx patients, $35.4 \%$ of the admissions over the 18-month period occurred between mid-March to May 2020 (Fig. 1). By May 2020, Hispanic/Latinx patients represented 21.7\% (69 total patient admissions, with 15 patients being Hispanic/Latinx) of COVID-19 ICU admissions. By contrast, during the pre-COVID-19 period, Hispanic/Latinx patients represented $2.34-5.27 \%$ of monthly ICU admissions (Fig. 1). However, there was no difference in age between Hispanic/Latinx ICU patients admitted before ( $48.46 \pm 13.07$ years old) and after $(49.67 \pm 19.50$ years old $)(p=0.75)$ COVID- 19 . There 
was no difference in Hispanic/Latino male patient admissions either, as they continued to represent the majority sex admitted to the ICU: 34 patients (66.67\%) prior to COVID-19 and 21 (75.00\%) during COVID$19(p=0.61)$. There was no difference in ICU length of stay pre- versus post-COVID-19 $(p=0.14)$ and no difference in mortality between the two time periods (4 deaths of Hispanic/Latinx patients prior to COVID19 ICU admissions as compared to 1 death during COVID-19 ICU admissions) $(p=0.79)$.

During COVID-19 ICU admissions, Hispanic/Latinx patients were younger ( $48.46 \pm 13.07$ years old) as compared to non-Hispanic/Latinx patients $(63.25 \pm 16.29$ years old $)(p<0.01)$ and predominantly male $(p$ $=0.04)$. However, deaths occurred at a greater rate in non-Hispanic/Latinx patients $(28.02 \%)$ as compared to Hispanic/Latinx patients $(3.57 \%)(p=0.01)$. Table 2 further emphasizes differences between race and ethnicity outcomes.

Table 2

A break down between ethnicity and race during the COVID-19 pandemic in a Baltimore City community hospital.

\begin{tabular}{|lllll|}
\hline & $\begin{array}{l}\text { Hispanic/Latinx } \\
(\mathbf{N}=\mathbf{2 8})\end{array}$ & $\begin{array}{l}\text { Non- } \\
\text { Hispanic/Latinx } \\
\text { African American } \\
\mathbf{N = 7 4 )}\end{array}$ & $\begin{array}{l}\text { Non-Hispanic/Latinx } \\
\text { White } \\
(\mathbf{N}=112)\end{array}$ & $\begin{array}{l}\mathbf{p}- \\
\text { value }\end{array}$ \\
\hline Age (years) & $48.46 \pm 13.07$ & $57.95 \pm 16.63$ & $64.95 \pm 15.96$ & $<0.01$ \\
\hline Male (\%) & $21(75.00)$ & $44(59.5)$ & $55(49.11)$ & 0.03 \\
\hline $\begin{array}{l}\text { ICU Length of Stay } \\
\text { (days) }\end{array}$ & $\begin{array}{l}3.00(1.75, \\
8.25)\end{array}$ & $4.00(1.25,7.75)$ & $2.00(1.00,4.25)$ & 0.08 \\
\hline \begin{tabular}{l} 
Death (\%) \\
\hline
\end{tabular} & $1(3.57)$ & $23(31.08)$ & $29(25.89)$ & $<0.01$ \\
\hline
\end{tabular}

\section{African American Patients}

Among African American patients, $13.5 \%$ of the admissions over the 18-month period occurred between mid-March to May 2020 (Fig. 1). By May 2020, 41 African American patients (59.42\%) accounted for the 69 total patient admissions to a COVID-19 ICU. By contrast, during the pre-COVID-19 period, monthly admissions to the MICU or CICU among African American patients ranged from 37.23-42.28\% (Fig. 1), never achieving the majority of admissions by race in either intensive care unit. There was no statistically significant difference in age between African American patients admitted before ( $58.96 \pm 15.91$ years old) and after ( $57.95 \pm 16.63$ years old) ( $p=0.56$ ) COVID-19. There was a statistically significant difference in ICU length of stay pre-COVID-19 and during COVID-19 ICU among African American patients. Pre-COVID19 , the median ICU length of stay was 3.00 days (IQR 1.00, 6.00), while during COVID-19 the ICU length of stay was 4.00 days (IQR $1.25,7.75)(p=0.04)$. Further, there was a statistically significant difference in mortality in African American pre-COVID-19 ICU admissions (113 patients [23.89\%]) as compared to African Americans during COVID-19 ICU admission (74 patients [31.08\%]) $(p=0.04)$. 
During COVID-19 ICU admissions, African American patients were younger (57.95 \pm 16.63 years old) as compared to White patients $(64.95 \pm 15.96$ years old $)(p<0.01)$. Further, African Americans had more ICU days as compared to Whites $(p=0.02)$. There was no difference in mortality between the two races during the COVID-19 ICU admissions over the 3-month period.

\section{Elderly}

Among elderly patients, $12.4 \%$ of the admissions over the 18 -month period occurred between mid-March to May 2020. There was no statistically significant difference in age between elderly patients admitted before (75.42 \pm 8.46 years old) and after $(75.60 \pm 8.60$ years old $)(p=0.84)$ COVID-19. There was no difference in sex between pre-COVID-19 admissions and COVID-19 admissions in the elderly $(p=0.25)$. Prior to COVID-19 admissions, White elderly accounted for $573(71.71 \%)$ of total elderly patients, while among COVID-19 admissions White elderly accounted for $61.61 \%$ (61 patients $(p=0.0495)$. Finally, there was a non-statistically significant increase in mortality in the elderly during COVID-19 admission (37 patients $(37.37 \%))$ as compared to pre-COVID-19 admissions (228 patients $(28.53 \%))(p=0.82)$. When evaluating elderly patients by race, there was a statistically significant increase in mortality in African American elderly patients during COVID-19 admissions (23 patients (31.08\%)) as compared to pre-COVID19 admissions (113 patients $(19.82 \%)(p=0.02)$. There were no deaths in elderly Hispanic/Latino patients (6 patients total) during COVID-19 ICU admissions.

\section{Discussion}

During the first three months of admissions to a COVID-19 ICU at an urban, academic, community hospital, there was a rise in admissions among Hispanic/Latinx and African-American patients, while non-Hispanic/Latinx and White patient admissions declined over the recent 3-month period. Compared to non-Hispanic/Latinx patients admitted to the ICU, Hispanic/Latinx admitted to the COVID-19 ICU were younger. As expected given the age differential, Hispanic/Latinx had greater survival rates compared to non-Hispanic/Latinx. African American patients had worse mortality outcomes during the pandemic as compared to non-COVID-19 ICU admissions, especially elderly African American patients. Overall, this shift in both admissions and outcomes based on race and ethnicity was a significant change during the COVID-19 pandemic for this community hospital in comparison to the previous year's ICU admissions.

Disparities in critical care diagnoses and admissions have been well documented. ${ }^{5,6,14}$ And in the case of the COVID-19 pandemic, disparities continue to exist in the proportion of minorities that are impacted by COVID-19. ${ }^{15}$ In the analysis by Price-Haywood et al, they found a disproportionate impact on African Americans with COVID-19: $76.9 \%$ of the patients hospitalized were African American, and $70.6 \%$ of the patients who died were African American. ${ }^{16}$ The data reviewed spanned the first 6-weeks of the public health crisis of COVID-19 in Louisiana (March 1 to April 11, 2020). Such a disproportionate impact was also seen in African Americans in our hospital's admissions. However, our data showcases that the pandemic had differential effects, increasing admissions of African American patients while simultaneously decreasing admissions of White patients. This finding is new, as evident by our review of 
critical care admissions the previous years, which were consistently stable for both African Americans and Whites, as well as the same pattern for Hispanic/Latinx patients versus non-Hispanic/Latinx patients.

Optimization of regional ICU utilization has been a priority during the COVID-19 pandemic. ${ }^{17}$ Using timelapsed, sociodemographic ICU admission may be part of the strategies to identify communities disproportionately impacted by COVID-19 and begin to attenuate such a disparity. This would parallel other interventions, where insight into ICU admissions may result in community efforts to mitigate these severe diseases. For instance, Victor et al demonstrated a community intervention to impact blood pressure control on non-Hispanic African American men by partnering with barbershops in Los Angeles; ${ }^{18}$ such an effort is warranted as this population has the highest rate of hypertension-related critical care admissions $^{19}$. ICU-level information reaffirms the need to promote testing and identify resources to facilitate isolation and quarantine for African Americans ${ }^{16,20,21}$ and Hispanic/Latinx ${ }^{20-22}$ who are experiencing COVID-19-related health disparities. Such strategies will require partnerships with neighborhood leaders to leverage community-based resources and venues to effect these collaborative changes, as well as structural changes at levels of policy and advocacy.

This study has several limitations. First, we did not evaluate more granular clinical data, such as severity of symptoms on presentation and pre-existing conditions. Second, we did not investigate what COVID-19 related syndromes warranted ICU admissions (e.g. sepsis, ARDS, heart failure). While these limitations are important to address in future studies and are necessary overall to understand the complete clinical presentation of COVID-19, we believe such information was not as relevant for implementing immediate community engagement initiatives towards populations impacted disproportionately by the pandemic. Finally, this information is relevant for our community hospital based in an urban region; it is unclear if similar findings would occur in community hospitals serving rural regions. This should be investigated in order to understand urban versus rural differences on populations as related to COVID-19 incidence and ICU admissions.

In the first months of the pandemic, there was a significant shift in persons admitted to an urban community hospital's ICU with regards to socio-demographics. An increase in minority populations based on race and ethnicity was evident in COVID-19 ICUs, while non-minority populations saw a decrease in ICU admissions. While much has been said regarding the disproportionate impact in minorities due to COVID-19, we have shown, using time-lapse evaluations, that this impact is significantly different than pre-pandemic ICU admission trends. Therefore, as strategies are discussed to curb the incidence of COVID-19 in minority populations, monitoring if such a reduction results in a decrease in ICU admissions should be emphasized as a public health priority.

\section{Abbreviations}

Acute Respiratory Distress Syndrome, ARDS; Severe Acute Respiratory Syndrome-Coronavirus-2, SARSCoV-2; Coronavirus Infectious Disease 2019, COVID19; intensive care unit, ICU; Johns Hopkins Bayview 
Medical Center, JHBMC; Persons Under Investigation, PUl; analysis of variance, ANOVA

\section{Declarations}

Ethics approval and consent to participate. The study was approved by Institutional Review Board at Johns Hopkins School of Medicine and all actions undertaken by the authors were in accordance with the Declaration of Helsinki.

Consent for publication. Not applicable as data is not of an individual person that could be identified based on the above findings.

Availability of data and material. The datasets used and/or analysed during the current study are available from the corresponding author on reasonable request.

Competing interests. The authors declare that they have no competing interests.

Funding. Not applicable as funding was not utilized to perform this analysis and review.

Authors' contributions. PG, ESC, and SHG were involved in conceptualization, data analyses, manuscript writing (original draft), and manuscript editing. KRP, SC, SS, and VJ were involved in formal analyses and manuscript editing. TT, FHB, and SS were involved in conceptualization and data analyses. JM was involved in data curation and manuscript editing.

Acknowledgements. Authors would like to acknowledge the staff of JHBMC involved in helping care for critically ill patients during the early months of the pandemic.

\section{References}

1. Chang DW, Shapiro MF. Association Between Intensive Care Unit Utilization During Hospitalization and Costs, Use of Invasive Procedures, and Mortality. JAMA Intern Med. 2016;176(10):1492-1499.

2. Mayr FB, Yende $S$, Linde-Zwirble WT, et al. Infection rate and acute organ dysfunction risk as explanations for racial differences in severe sepsis. JAMA. 2010;303(24):2495-2503.

3. Goodwin AJ, Nadig NR, McElligott JT, Simpson KN, Ford DW. Where You Live Matters: The Impact of Place of Residence on Severe Sepsis Incidence and Mortality. Chest. 2016;150(4):829-836.

4. Kar P, Jones KL, Horowitz M, Deane AM. Management of critically ill patients with type 2 diabetes: The need for personalised therapy. World J Diabetes. 2015;6(5):693-706.

5. Soto GJ, Martin GS, Gong MN. Healthcare disparities in critical illness. Crit Care Med. 2013;41(12):2784-2793.

6. Erickson SE, Vasilevskis EE, Kuzniewicz MW, et al. The effect of race and ethnicity on outcomes among patients in the intensive care unit: a comprehensive study involving socioeconomic status and resuscitation preferences. Crit Care Med. 2011;39(3):429-435.

7. Wang HE, Shapiro NI, Griffin R, Safford MM, Judd S, Howard G. Chronic medical conditions and risk of sepsis. PLoS One. 2012;7(10):e48307.

8. Moss M, Mannino DM. Race and gender differences in acute respiratory distress syndrome deaths in the United States: an analysis of multiple-cause mortality data (1979-1996). Crit Care Med. 
2002;30(8):1679-1685.

9. Galiatsatos P, Kachalia A, Belcher HME, et al. Health equity and distributive justice considerations in critical care resource allocation. Lancet Respir Med. 2020;8(8):758-760.

10. Grasselli G, Greco M, Zanella A, et al. Risk Factors Associated With Mortality Among Patients With COVID-19 in Intensive Care Units in Lombardy, Italy. JAMA Intern Med. 2020.

11. Grasselli G, Zangrillo A, Zanella A, et al. Baseline Characteristics and Outcomes of 1591 Patients Infected With SARS-CoV-2 Admitted to ICUs of the Lombardy Region, Italy. JAMA. 2020.

12. Baltimore City Health Department. Neighborhood Health Profile Reports.,https://health.baltimorecity.gov/neighborhoods/neighborhood-health-profile-reports. :Accessed 10 July 2020.

13. Knezevich A. First Baltimore City case of coronavirus confirmed. Baltimore Sun. 2020;https://www.baltimoresun.com/coronavirus/bs-md-coronavirus-city-first-case-20200315hp54laqpgjhilgyx6thqht|3ra-story.html. :Accessed 30 July 2020.

14. Martin GS, Mannino DM, Eaton S, Moss M. The epidemiology of sepsis in the United States from 1979 through 2000. N Engl J Med. 2003;348(16):1546-1554.

15. Martinez DA, Hinson JS, Klein EY, et al. SARS-CoV-2 Positivity Rate for Latinos in the BaltimoreWashington, DC Region. JAMA. 2020.

16. Price-Haywood EG, Burton J, Fort D, Seoane L. Hospitalization and Mortality among Black Patients and White Patients with Covid-19. N Engl J Med. 2020;382(26):2534-2543.

17. Maves RC, Downar J, Dichter JR, et al. Triage of Scarce Critical Care Resources in COVID-19 An Implementation Guide for Regional Allocation: An Expert Panel Report of the Task Force for Mass Critical Care and the American College of Chest Physicians. Chest. 2020;158(1):212-225.

18. Victor RG, Lynch K, Li N, et al. A Cluster-Randomized Trial of Blood-Pressure Reduction in Black Barbershops. N Engl J Med. 2018;378(14):1291-1301.

19. Benjamin EJ, Blaha MJ, Chiuve SE, et al. Heart Disease and Stroke Statistics-2017 Update: A Report From the American Heart Association. Circulation. 2017;135(10):e146-e603.

20. Kirby T. Evidence mounts on the disproportionate effect of COVID-19 on ethnic minorities. Lancet Respir Med. 2020;8(6):547-548.

21. Selden TM, BerdahI TA. COVID-19 And Racial/Ethnic Disparities In Health Risk, Employment, And Household Composition. Health Aff (Millwood). 2020:101377hlthaff202000897.

22. Webb Hooper M, Napoles AM, Perez-Stable EJ. COVID-19 and Racial/Ethnic Disparities. JAMA. 2020.

\section{Figures}



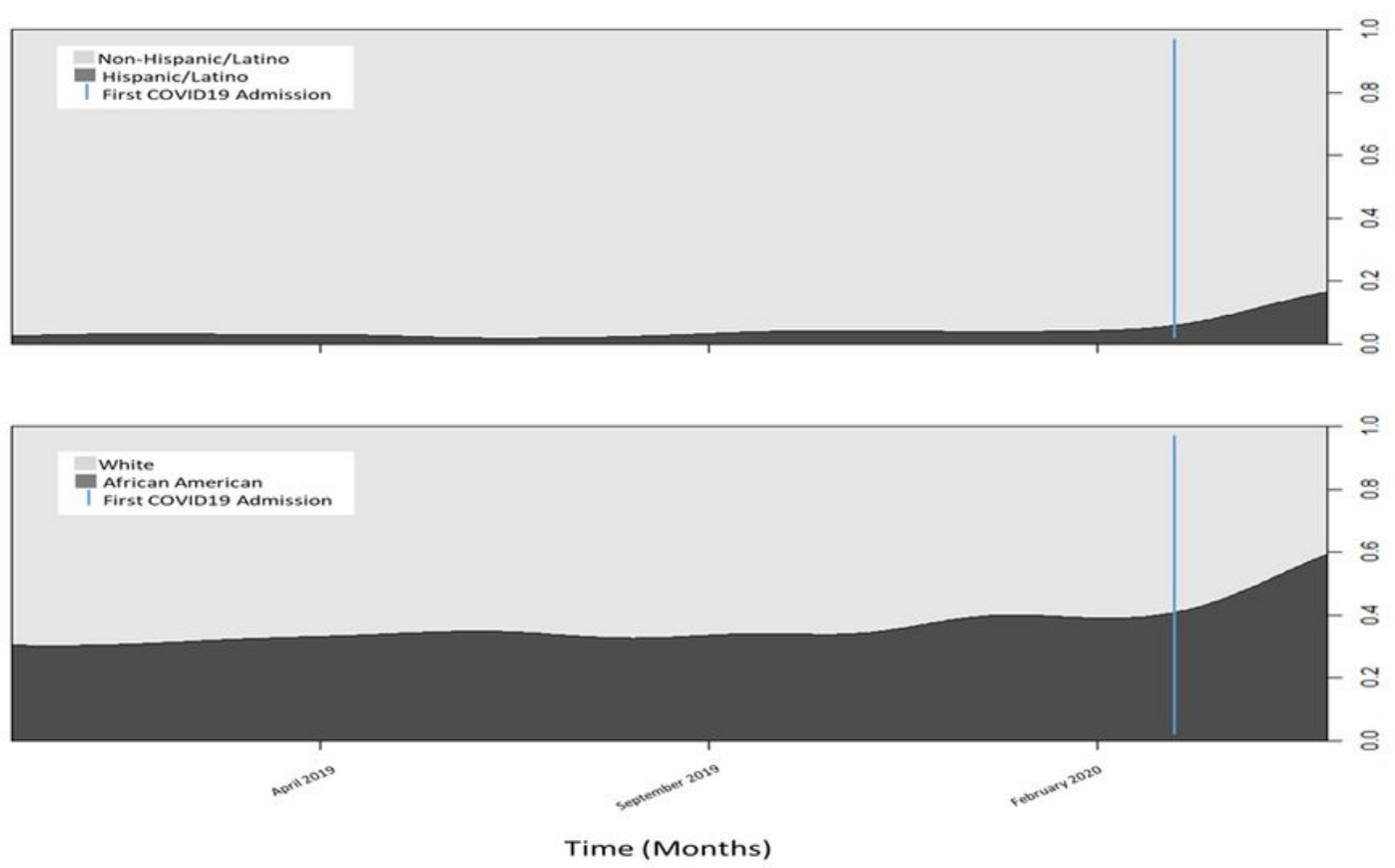

\section{Figure 1}

Comparion of intensive care unit admissions by minority populations before and after SARS-CoV- 2 admissions.
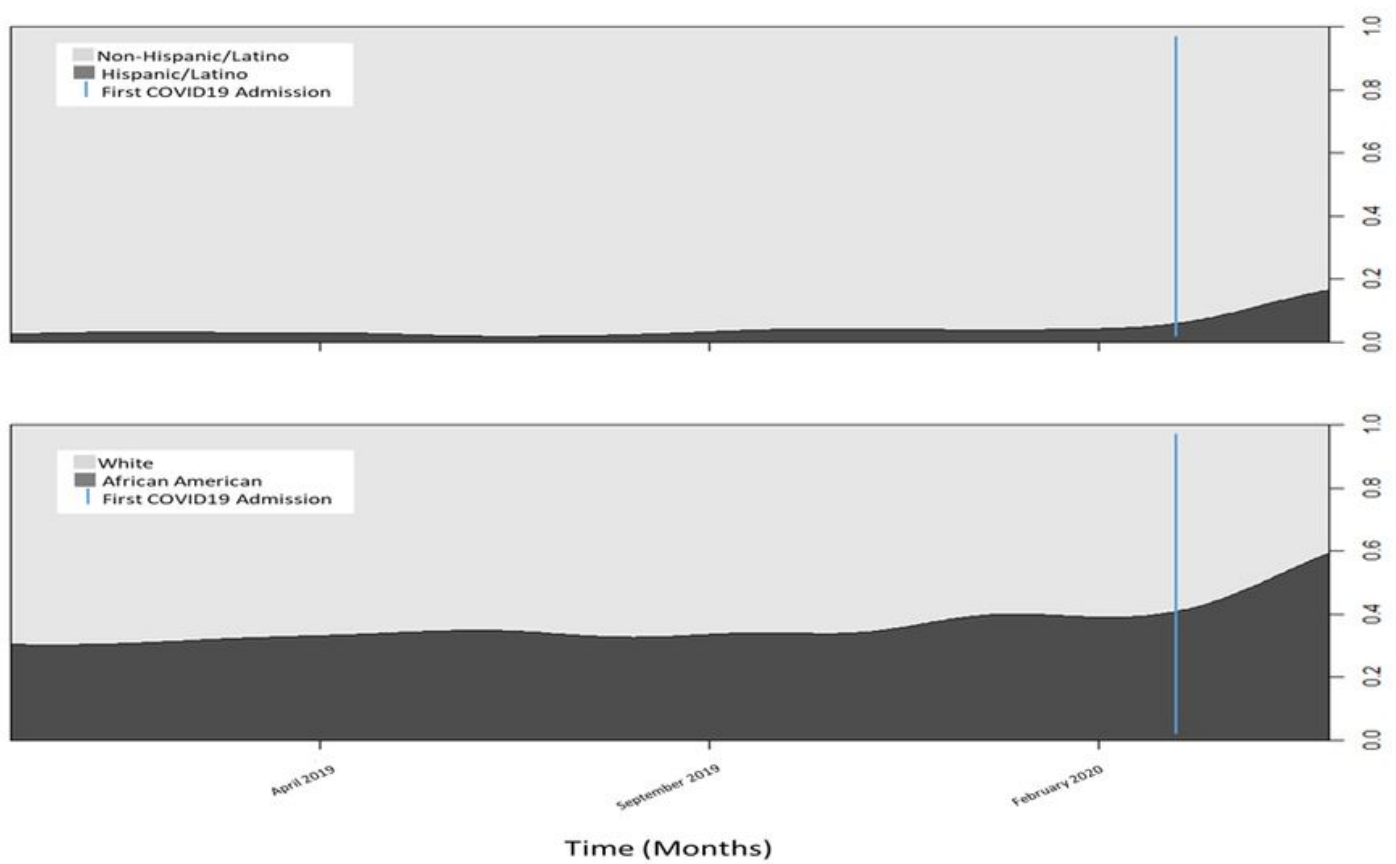


\section{Figure 1}

Comparion of intensive care unit admissions by minority populations before and after SARS-CoV-2 admissions. 